\title{
Simplified selection criteria for patients with longer or unknown time to treatment predict good outcome after mechanical thrombectomy
}

\author{
Simon Nagel, ${ }^{\oplus 1}$ Christian Herweh, ${ }^{2}$ Johannes Alex Rolf Pfaff, ${ }^{\oplus}{ }^{2}$ Simon Schieber, ${ }^{1}$ \\ Silvia Schönenberger, ${ }^{1}$ Markus A Möhlenbruch, ${ }^{2}$ Martin Bendszus, ${ }^{2}$ \\ Peter Arthur Ringleb ${ }^{1}$
}

\begin{abstract}
- Additional material is published online only. To view please visit the journal online (http://dx.doi.org/10.1136/ neurintsurg-2018-014347).

${ }^{1}$ Department of Neurology, University of Heidelberg, Heidelberg, Germany ${ }^{2}$ Department of Neuroradiology, University Hospital Heidelberg, Heidelberg, Germany
\end{abstract}

\section{Correspondence to} Dr Simon Nagel, Department of Neurology, University Hospital Heidelberg, INF 400, Heidelberg 69120, Germany; simon.nagel@ med.uni-heidelberg.de

SN and $\mathrm{CH}$ contributed equally.

Received 14 August 2018 Revised 26 September 2018 Accepted 2 October 2018 Published Online First 27 October 2018

Check for updates

(C) Author(s) (or their employer(s)) 2019. No commercial re-use. See rights and permissions. Published by BMJ.

To cite: Nagel S, Herweh $C$, Pfaff JAR, et al.

J Neurolntervent Surg

2019:11:559-562.

\section{ABSTRACT}

Objective To identify simplified selection criteria for mechanical thrombectomy (MT) in longer and unknown time windows.

Methods Patients with large vessel occlusion (LVO) in the anterior circulation who underwent MT between January 2014 and November 2017 were identified from the local registry. Patients were selected for analysis if they met the current guideline recommendation for MT treatment except for time window (HERMES-like) and were divided according to time they were last seen well (LSW): LSW $<6$ hours or LSW $>6$ hours before MT. The primary endpoint, good outcome, was modified Rankin scale score $0-2$ on day 90 . Safety outcomes were mortality on day 90 and symptomatic intracranial hemorrhage $(\mathrm{sICH})$. Univariate and multivariate analysis were performed for good outcome in HERMES-like patients.

Results In total, 752 patients were identified and 390 patients (51.9\%) fulfilled the HERMES-like criteria. Despite differences in baseline parameters, more diffusion-weighted imaging (DWI) $433.9 \%$ vs $11.3 \%$, $\mathrm{p}<0.001)$ and fewer cases of thrombolysis $(32.7 \%$ vs $77 \%, p<0.001)$, patients LSW $>6$ hours $(n=107)$ did not differ in the primary and secondary endpoints: good outcome ( $44.9 \%$ vs $44.9 \%, p=1.0)$, mortality ( $14 \%$ vs $15.2 \%, p=0.87)$, and sICH (5.6\% vs $6 \%, p=1.0)$. After multivariate regression analysis, independent predictors of good outcome remained: age, $\mathrm{OR}=0.96(95 \% \mathrm{Cl}$ 0.95 to 0.98$)$; National Institutes of Health Stroke Scale score, OR=0.92 (95\% Cl 0.89 to 0.96); Alberta Stroke Programme Early CT Score (ASPECTS), OR=1.26 (95\% Cl 1.06 to 1.49); general anesthesia, $O R=0.2(95 \% \mathrm{Cl} 0.04$ to 0.99$)$, and successful recanalization, $O R=12(95 \%$ $\mathrm{Cl} 4.7$ to 30.5); but not treatment time and DWI or CT perfusion at baseline.

Conclusion Patients with proven LVO in unknown and longer time windows may be selected for MT based on ASPECTS and clinical criteria.

\section{INTRODUCTION}

Evidence for the efficacy and safety of mechanical thrombectomy (MT) in patients with large vessel occlusion (LVO) of the anterior circulation is overwhelming. The HERMES (Highly Effective Reperfusion evaluated in Multiple Endovascular Stroke trials) collaborators performed two meta-analyses of five randomized trials, which showed that the proportion of patients who achieved a good outcome as defined by 0 to 2 points on the modified Rankin scale (mRS) was $46 \%$ after 90 days, and the odds of better disability outcomes at 90 days with the endovascular group retained statistically significant through 7 hours and 18 min. $^{12}$ Recently, the DAWN (Clinical Mismatch in the Triage of Wake Up and Late Presenting Strokes Undergoing Neurointervention With Trevo) trial and the DEFUSE 3 (Endovascular Therapy Following Imaging Evaluation for Ischemic Stroke 3) also provided evidence that MT is effective and safe, as compared with best medical treatment, in patients who were last seen well from 6 and up to 24 hours before treatment. $^{3}{ }^{4}$ However, inclusion in both trials was based on automated estimation of infarct core (and mismatch) by either CT perfusion (CTP) or diffusion-weighted MR imaging (DWI). The most recent guidelines now recommend applying the DAWN and DEFUSE 3 imaging criteria (perfusion imaging-based) to select patients in unknown and longer time windows. ${ }^{5}$ We aimed to test in a large monocentric and prospectively collected cohort whether patients with LVO may be successfully selected for MT based on the Alberta Stroke Programme Early CT Score (ASPECTS) and clinical criteria only, irrespective of time.

\section{METHODS}

We searched our prospective database, the Heidelberg Recanalization Registry (HeiReKa), for patients with ischemic strokes due to LVO in the anterior circulation who had received MT up to 24 hours or unknown time from onset between 2014 and November 2017 (4 years). During this time our local imaging protocol requirements and treatment recommendations varied but included at least a non-contrast-enhanced CT (NCCT) scan and a CT angiogram for baseline imaging (online supplementary material). CTP and magnet resonance perfusion parameters included time to peak, cerebral blood volume, and cerebral blood flow and were calculated using the software provided by the manufacturer of the CT and MR apparatus (Siemens, Erlangen, Germany). Mismatch on perfusion imaging between time to peak and cerebral blood volume maps or DWI was only semiquantitatively assessed and defined as described in the 
European Cooperative Acute Stroke Study (ECASS) IV trial protocol: penumbral imaging with a perfusion volume (PWI) to infarct core (DWI) ratio of 1.2 , and a perfusion lesion minimum volume of $20 \mathrm{~mL}^{6}{ }^{7}$ Treatment decisions were made by the local team (stroke physician and neuroradiologist on call), but generally MT was recommended according to our local standard operating procedures following national and international guidelines (online supplementary material).

Between April 2014 and February 2016 eligible patients were included in our single-center randomized sedation trial SIESTA ${ }^{8}$ comparing general anesthesia with conscious sedation; thereafter, the primary mode of sedation was conscious sedation. Recanalization was assessed using the Thrombolysis in Cerebral Infarction scale by an experienced neuroradiologist, and successful recanalization was defined as $\geq 2 \mathrm{~b}$. The ASPECTS score was calculated by e-ASPECTS (www.brainomix.com) on baseline CT. e-ASPECTS has been being used as a research tool since 2014 and since 2015 as a decision support tool in routine clinical practice in our institution. In both cases, the e-ASPECTS results were available to the treating physician. The non-inferior performance of e-ASPECTS for neuroradiologists as well as its clinical utility has been described previously. ${ }^{9}{ }^{10}$ If MRI was performed, ASPECTS was determined by two investigators (SN and JARP), who in 'HERMES-like' patients beyond 6 hours also reviewed all CTP and MRI images for the presence of mismatch as defined above.

The outcome was measured with the mRS after 90 days and obtained through rehabilitation reports, outpatient assessments, or a standardized interview by an unblinded investigator. The primary endpoint 'good clinical outcome' was defined as mRS score 0-2. Safety endpoints were mortality after 90 days and symptomatic intracranial hemorrhage ( $\mathrm{sICH}$ ) defined according to ECASS II. $^{11}$ As recommended in the guidelines, patients were included in the analysis if they met the following criteria for patient selection within 6 hours from onset ${ }^{5}$ and labeled as 'HERMES-like': (i) premorbid mRS 0-1, (ii) ASPECTS $\geq 6$ (either on CT or DWI), and (iii) National Institutes of Health Stroke Scale (NIHSS) score $\geq 6$. Patients who underwent MT twice within the period were excluded.

Patients were then divided into two groups according to the time last seen well (LSW): LSW $<6$ hours-that is, within 6 hours, and LSW $>6$ hours-that is, beyond 6 hours, before initiating MT. Nominal variables are described as frequencies, ordinal variables as median (IQR), and continuous variables as mean \pm SD. Differences between groups were assessed with Fisher's exact test, $\chi^{2}$ test, Mann-Whitney test, or t test as appropriate, and a $\mathrm{p}$ value of 0.05 was considered statistically significant. Univariate and multivariate analyses for the primary endpoint 'good outcome' were performed. All variables with $\mathrm{p}<0.05$ in the univariate analysis were included in a forward stepwise logistic regression model. Odds ratios (ORs) are described with $95 \%$ confidence intervals. HeiReKa and analyses within were approved by the ethics committee of Heidelberg University (S-325/2015). Owing to the monocentric and retrospective nature of the study, informed consent of the patients was waived.

\section{RESULTS}

In total, 752 patients received an MT for LVO in the anterior circulation within 24 hours after last being seen well in the given time frame. Three hundred and ninety patients (51.9\%) fulfilled the HERMES-like guidelines criteria and were compared according to treatment time (figure 1/table 1). Data for the excluded patients $(n=362,48.1 \%)$ are shown in the online

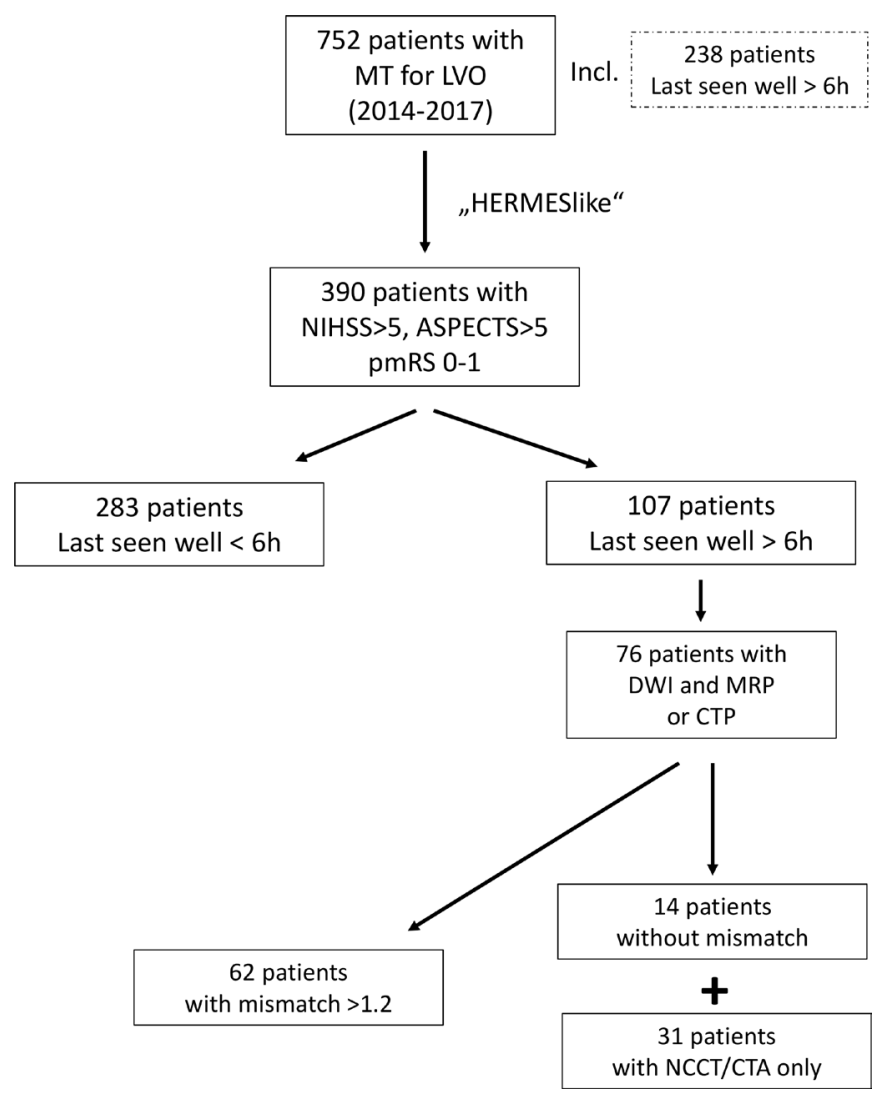

Figure 1 Flowchart of patients. ASPECTS, Alberta Stroke Programme Early CT Score; CTA, CT angiography; CTP, CT perfusion; DWI, diffusionweighted imaging; LVO, large vessel occlusion; MRP, magnetic resonance perfusion; MT, mechanical thrombectomy; NCCT, noncontrast-enhanced CT; NIHSS, National Institute of Health Stroke Scale; pmRS, premorbid modified Rankin Scale.

supplementary material. Interestingly, patients did not differ in the primary and secondary endpoints: good outcome was reached in $44.9 \%$, irrespective of time to treatment (figure 2), and sICH was also low and comparable in the two groups $(6 \%$ vs $5.6 \%, \mathrm{p}=1.0)$. Patients in the LSW $>6$ hours group, however, differed in important baseline, imaging, and treatment variables, such as gender, wake-up stroke, occlusion type, imaging modality, including perfusion imaging, and ASPECTS (table 1). They were also less often treated with IV thrombolysis and by definition had much longer onset to treatment times than those patients with witnessed onset; rates of successful recanalization were similarly high in the two groups (table 1). In patients with longer time windows (LSW $>6$ hours) examined with MRI or CTP, mismatch was present in $81.6 \%(62 / 76)$; in $18.4 \%(14 / 76)$ mismatch was smaller than $20 \%$ or the perfusion imaging could not be analyzed (for technical reasons). Comparing the primary endpoint good outcome in HERMES-like and LSW > 6 hours patients with mismatch $(62 / 107)$ with those without mismatch or core imaging only $(45 / 107)$, we still found no relevant difference $(46.8 \%$ vs $42.9 \%, \mathrm{p}=0.69)$.

The only factors that were significantly associated with good outcome in univariate analysis were premorbid disability (mRS 1), age, NIHSS, and ASPECTS at baseline, general anesthesia, and recanalization (table 2), but not the application of MR or CT perfusion imaging. After multivariate regression analysis, independent predictors of good outcome remained: younger age $\mathrm{OR}=0.96$ per year $(95 \%$ CI 0.95 to 0.98$)$, lower NIHSS per point OR=0.92 (95\% CI 0.88 to 0.96), higher ASPECTS per 


\begin{tabular}{|c|c|c|c|}
\hline Parameters & $\begin{array}{l}\text { LSW }<6 \text { hours } \\
(n=283)\end{array}$ & $\begin{array}{l}\text { LSW }>6 \text { hours } \\
(n=107)\end{array}$ & $P$ values \\
\hline \multicolumn{4}{|l|}{ Clinical baseline data } \\
\hline Age (years) & $74(18)$ & $74(17)$ & $0.62^{*}$ \\
\hline Male sex (\%) & $161(56.9)$ & $46(43)$ & $0.017 \dagger$ \\
\hline Unwitnessed/wake-up onset (\%) & $2(0.7)$ & $90(84.1)$ & $<0.001 \dagger$ \\
\hline NIHSS (median) & $16(8)$ & $15(9)$ & $0.18^{*}$ \\
\hline $\begin{array}{l}\text { Premorbid disability, mRS score } \\
1(\%)\end{array}$ & $109(38.5)$ & $44(41.1)$ & $0.64 \dagger$ \\
\hline \multicolumn{4}{|l|}{ Risk factors } \\
\hline High blood pressure (\%) & $189(70)$ & $72(67.3)$ & $0.62 \dagger$ \\
\hline Diabetes (\%) & $54(19.1)$ & $23(21.5)$ & $0.67 \dagger$ \\
\hline High cholesterol (\%) & $89(31.4)$ & $33(30.8)$ & $1.0 \dagger$ \\
\hline Current smoking (\%) & $39(13.8)$ & $16(15)$ & $0.75 t$ \\
\hline Known atrial fibrillation (\%) & $74(26.1)$ & $26(24.3)$ & $0.83 \dagger$ \\
\hline $\begin{array}{l}\text { Newly diagnosed atrial } \\
\text { fibrillation (\%) }\end{array}$ & $51(18)$ & $22(20.6)$ & \\
\hline Previous stroke (\%) & 39 (13.8) & $15(14)$ & $1.0 \dagger$ \\
\hline Coronary heart disease (\%) & $80(28.3)$ & $18(16.8)$ & $0.026 t$ \\
\hline \multicolumn{4}{|l|}{ Site of occlusion } \\
\hline Proximal CA (\%) & $10(3.5)$ & $10(9.3)$ & $0.02 \dagger$ \\
\hline Tandem (\%) & $36(12.7)$ & $4(3.7)$ & \\
\hline Distal CA (\%) & $57(20.1)$ & $24(22.4)$ & \\
\hline M1 (\%) & $125(44.2)$ & $48(44.9)$ & \\
\hline M2 (\%) & $55(19.4)$ & $21(19.6)$ & \\
\hline \multicolumn{4}{|l|}{ Brain imaging } \\
\hline MRI (\%) & $32(11.3)$ & $44(41.1)$ & $<0.001 \dagger$ \\
\hline CTP $(\%)$ & $147(51.9)$ & $32(30)$ & $<0.001 \dagger$ \\
\hline ASPECTS (median) & $9(2)$ & $8(2)$ & $<0.001 *$ \\
\hline \multicolumn{4}{|l|}{ Procedural data } \\
\hline IV thrombolysis (\%) & $218(77)$ & $35(32.7)$ & $<0.001 \dagger$ \\
\hline LSW to treatment time $(\mathrm{min})$ & $133 \pm 70$ & $563 \pm 206$ & $<0.001 \neq$ \\
\hline Door-to-vessel time (DTV, min) & $84 \pm 77$ & $90 \pm 36$ & $0.25 \ddagger$ \\
\hline General anesthesia (\%) & $82(29)$ & $32(30)$ & $0.35 t$ \\
\hline Recanalization (\%) & $239(84.5)$ & $95(88)$ & $0.33 \dagger$ \\
\hline \multicolumn{4}{|l|}{ Outcome } \\
\hline mRS score 0-2 (\%) & $127(44.9)$ & $48(44.9)$ & $1.0 \dagger$ \\
\hline mRS score $6(\%)$ & $43(15.2)$ & $15(14)$ & $0.87 \dagger$ \\
\hline sICH (\%) & $17(6)$ & $6(5.6)$ & $1.0 \dagger$ \\
\hline
\end{tabular}

*Mann-Whitney test.

$+\chi^{2} /$ Fisher test.

ft Test.

ASPECTS, Alberta Stroke Program Early CT Score; CA, carotid artery; CTP, CT perfusion; LSW, last seen well; mRS, modified Rankin Scale; NIHSS, National Institutes of Health Stroke Scale; M1/M2, M1 resp. M2 segment of the middle cerebral artery; $\mathrm{sICH}$, symptomatic intracerebral hemorrhage.

point $\mathrm{OR}=1.25$ (95\% CI 1.06 to 1.49$)$, avoiding general anesthesia $\mathrm{OR}=0.2(95 \% \mathrm{CI} 0.04$ to 0.99$)$, and successful recanalization $\mathrm{OR}=12(95 \% \mathrm{CI} 4.7$ to 30.5$)$.

\section{DISCUSSION}

Our study, which to the best of our knowledge was performed in the largest non-randomized cohort of patients in routine clinical

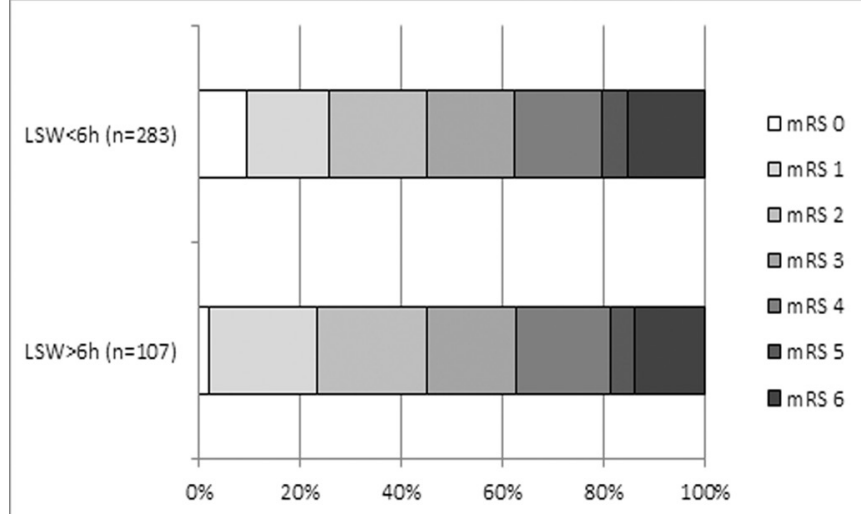

Figure 2 Clinical outcome after 90 days on the modified Rankin Scale $(m R S)$ in HERMES-like patients $(n=390)$. LSW, last seen well.

practice undergoing MT for LVO in the anterior circulation in unknown or longer time windows ( $n=238$ in total), found no difference in outcome compared with those patients treated within 6 hours after last having been seen well. In HERMESlike patients $(n=390)$ - that is, those fulfilling the current guideline criteria for treatment within 6 hours, rates of good outcome were $44.9 \%$ in patients LSW $<6$ hours and LSW $>6$ hours, being comparable to the HERMES meta-analysis ${ }^{1}$ and both DAWN and DEFUSE $3 .^{34}$ Both safety endpoints, mortality and sICH, were also similar and low in both groups. Importantly, the performance of MRI or CTP at baseline was not associated with outcome in our HERMES-like cohort, and the clinical outcome of patients with MRI and CTP and present mismatch was also similar to those without mismatch, or core imaging only (CT or DWI). The only independent predictors of good outcome were younger age, lower NIHSS, higher ASPECTS, conscious sedation, and successful recanalization.

The DAWN and DEFUSE 3 trials made an invaluable contribution to the field and trial inclusion was based on automated assessment by the RAPID software (www.ischemaview.com) of CT perfusion or DWI (which are not readily available in most, even comprehensive, stroke centers) highlighting a small infarct core. Of note, patients in DAWN were also required to have less than one-third infarction of the territory of the middle cerebral artery on NCCT and patients in DEFUSE 3 had to have an ASPECTS of $\geq 6$ at baseline imaging. ${ }^{34}$ Most interestingly, the rate of good outcome in the control groups of both DAWN and DEFUSE 3 was very low (15\%), probably due to less use of recombinant tissue plasminogen activator and less frequent spontaneous recanalization. Such a poor outcome rate in the control groups means that even a small treatment effect through performance of MT will become meaningful.

Aside from DAWN and DEFUSE 3, published data on patients with wake-up strokes or strokes in longer time windows undergoing MT are scarce. Although the reported outcome data do seem encouraging, the studies are compromised by exclusion of MT candidates if MT could not be started within 6 hours from last having been seen well ${ }^{12}$ and by considerably smaller sample sizes $\left(n=12^{13}\right.$ and $\left.n=22\right) .{ }^{14}$ The DAWN trial included $90 \%$ of patients who had had an unwitnessed stroke or stroke on awakening. In our cohort, almost $85 \%$ were included on this basis and the mean time to treatment among those with clear onset was around 9.5 hours. Earlier studies have already shown that clinical, neuroimaging, and etiopathogenic characteristics of patients with wake-up stroke are similar to those of patients with stroke of known onset. ${ }^{15} 16$ One study found 
Table 2 Variables significantly associated with the primary endpoint good outcome and their respective ORs in unadjusted and adjusted analysis

\begin{tabular}{lllcc}
\hline Variable & Unadjusted OR $(95 \% \mathrm{Cl})$ & P values & Adjusted OR (95\% Cl) & P values \\
\hline Age & $0.97(0.95$ to 0.98$)$ & $<0.001$ & $0.96(0.95$ to 0.98$)$ & $<0.001$ \\
\hline Premorbid mRS 1 & $0.6(0.4$ to 0.9$)$ & 0.015 & $0.75(0.47$ to 1.2$)$ & 0.23 \\
NIHSS & $0.94(0.9$ to 0.97$)$ & 0.001 & $0.92(0.88$ to 0.96$)$ & $<0.001$ \\
\hline ASPECTS & $1.26(1.07$ to 1.47$)$ & 0.005 & $1.25(1.06$ to 1.49$)$ & 0.008 \\
General anesthesia & $0.17(0.37$ to 0.74$)$ & 0.019 & $0.2(0.04$ to 0.99$)$ & 0.05 \\
Successful recanalization & $8.5(3.6$ to 20.4$)$ & $<0.001$ & $12(4.7$ to 30.5$)$ & $<0.001$ \\
\hline
\end{tabular}

ASPECTS, Alberta Stroke Programme Early CT Score; mRS, modified Rankin Scale; NIHSS, National Institutes of Health Stroke Scale.

that ischemic lesion volumes were similar in known onset and wake-up patients but higher in patients with indefinite onset strokes; the frequency of CTP mismatch, however, was similar across all patients. ${ }^{17}$ Examining different imaging-based selection paradigms for MT, Bouslama et al found that a perfusion imaging-based mismatch seemed to disqualify more patients from treatment without improving outcome as compared with clinical-core mismatch and clinical-ASPECTS mismatch. ${ }^{18}$ Interestingly, in our dataset, performance of MRI or CT perfusion imaging, although differing within patients LSW $<6$ hours and LSW $>6$ hours, did not predict outcome. Hence, our data indicate that it is probably not necessary to select patients with perfusion imaging in unknown and longer time windows as we showed that similar outcome and safety profiles can be achieved if patients are selected just by ASPECTS. Despite ASPECTS being reduced to the range of 6-10 in our HERMES-like cohort, it prevailed as independent predictor of outcome, thereby emphasizing its clinical utility. Indeed, ASPECTS assessment on NCCT in longer time windows is likely to be more accurate. ${ }^{19}$ Hence, like those in EXTEND-IA ${ }^{20}$ and SWIFT-PRIME, ${ }^{21}$ patients in DAWN and DEFUSE 3 are probably overselected - that is, some patients who were excluded owing to an 'unfavorable' perfusion imaging profile are also likely to benefit from MT. For DEFUSE 3 it was reported that around $30 \%$ of patients who consented did not fulfill the imaging criteria; for DAWN this number was not published. ${ }^{34}$

The other independent predictors of outcome such as age, NIHSS, and recanalization are well established and not surprising, highlighting the reproducibility of our results. The effect of general anesthesia on outcome, however, should be interpreted with caution since after completion of the SIESTA trial only very severely affected or agitated patients were treated under this mode of sedation in our department, thereby potentially biasing the results.

This study has some limitations; first, it is monocentric and retrospective and hence the comparative groups are misbalanced in some baseline variables, but data collection is prospective in HeiReKa and our results are comparable to those of recent randomized controlled trials and other large registries. HeiReKa does not contain data on patients who did not receive acute recanalization therapy; thus, we cannot report imaging profiles of patients who were evaluated for MT but thereafter did not receive treatment. Our supplementary analysis, however, shows that even in patients who received MT outside the current guideline (ie, despite lower ASPECTS and higher prestroke disability), treatment time was not a treatment effect modifier. Furthermore, MR and CT perfusion were only semiquantitatively assessed and not using automated software (which was done in DAWN and DEFUSE 3); however, the use of MRI and perfusion imaging showed no association with outcome.

\section{CONCLUSION}

In this large cohort of patients we demonstrate the utility of simple clinical (good baseline function, significant neurologic deficit) and imaging (high ASPECTS and CT angiography confirmation of large vessel occlusion) criteria in treatment selection for MT in late and unknown treatment time windows. Applying these criteria produced clinical outcomes and safety profiles equivalent to those of the cohort of patients undergoing earlywindow MT and to historical data from randomized controlled trials. No added usefulness was provided by perfusion imaging in this dataset. Further studies examining the role of perfusion in the more marginal cases (ie, non-HERMES-like) are warranted. We view these data as encouraging as we are attempting to expand the pool of candidates for late-window treatment and simplify the processes by which these patients are selected. These data also support the impression that real-world practice of MT may not necessarily need to mimic clinical trial design and execution to be reasonable.

Contributors All authors contributed substantially to the conception or design of the work; or the acquisition, analysis, or interpretation of data for the work AND in drafting the work or revising it critically for important intellectual content AND gave their final approval of the version to be published. All authors agreed to be accountable for all aspects of the work in ensuring that questions related to the accuracy or integrity of any part of the work are appropriately investigated and resolved.

Funding The authors have not declared a specific grant for this research from any funding agency in the public, commercial or not-for-profit sectors.

Competing interests Outside the published work, personal fees, travel support, speaker honoraria, or research grants were received from Acandis (MAM), Bayer (MB, PAR, SN), Brainomix (SN, CH), BMS Pfizer (MB, PAR, SN), Boehringer Ingelheim (MB, PAR, SN), Codman (MB, MAM), Medtronic (MAM, SN, MB), Deutsche Forschungsgesellschaft (MB, PAR), Guerbet (MB), Hopp foundation (MB), Microvention (MAM), Phenox (MAM), Roche (MB), Siemens (MB, JARP) and Stryker (CH, JARP, MAM), TEVA (MB), Vascular Dynamics (MB).

Patient consent Not required.

Ethics approval Heidelberg ethics committee.

Provenance and peer review Not commissioned; externally peer reviewed.

\section{REFERENCES}

1 Goyal M, Menon BK, van Zwam WH, et al. Endovascular thrombectomy after large-vessel ischaemic stroke: a meta-analysis of individual patient data from five randomised trials. Lancet 2016;387:1723-31.

2 Saver JL, Goyal M, van der Lugt A, et al. Time to treatment with endovascular thrombectomy and outcomes from ischemic stroke: a meta-analysis. JAMA 2016;316:1279-88.

3 Nogueira RG, Jadhav AP, Haussen DC, et al. Thrombectomy 6 to 24 hours after stroke with a mismatch between deficit and infarct. N Eng/ J Med 2018;378:11-21.

4 Albers GW, Marks MP, Kemp S, et al. Thrombectomy for stroke at 6 to 16 hours with selection by perfusion imaging. N Engl J Med 2018;378:708-18.

5 Powers WJ, Rabinstein AA, Ackerson T, et al. 2018 Guidelines for the early management of patients with acute ischemic stroke: a guideline for healthcare professionals from the American Heart Association/American Stroke Sssociation. Stroke 2018;49:e46-110. 
6 Amiri H, Bluhmki E, Bendszus M, et al. European cooperative acute stroke study-4: extending the time for thrombolysis in emergency neurological deficits ECASS-4: ExTEND. Int J Stroke 2016;11:260-7.

7 Hacke W, Furlan AJ, Al-Rawi Y, et al. Intravenous desmoteplase in patients with acute ischaemic stroke selected by MRI perfusion-diffusion weighted imaging or perfusion CT (DIAS-2): a prospective, randomised, double-blind, placebo-controlled study. Lancet Neurol 2009;8:141-50.

8 Schönenberger S, Uhlmann L, Hacke W, et al. Effect of conscious sedation vs general anesthesia on early neurological improvement among patients with ischemic stroke undergoing endovascular thrombectomy: a randomized clinical trial. JAMA 2016;316:1986-96

9 Nagel S, Sinha D, Day D, et al. e-ASPECTS software is non-inferior to neuroradiologists in applying the ASPECT score to computed tomography scans of acute ischemic stroke patients. Int J Stroke 2017;12:615-22.

10 Nagel S, Wang X, Carcel C, et al. Clinical utility of electronic Alberta Stroke Program early computed tomography score software in the ENCHANTED Trial Database. Stroke 2018;49:1407-11.

11 Hacke W, Kaste M, Fieschi C, et al. Randomised double-blind placebo-controlled trial of thrombolytic therapy with intravenous alteplase in acute ischaemic stroke (ECASS II). Second European-Australasian Acute Stroke Study investigators. Lancet 1998;352:1245-51.

12 Mourand I, Brunel H, Costalat V, et al. Mechanical thrombectomy in acute ischemic stroke: catch device. AJNR Am J Neuroradiol 2011;32:1381-5.
13 Konstas AA, Minaeian A, Ross IB. Mechanical thrombectomy in wake-up strokes: a case series Using Alberta Stroke Program Early CT Score (ASPECTS) for patient selection. J Stroke Cerebrovasc Dis 2017;26:1609-14.

14 Kuntze Söderqvist $\AA$, Andersson T, Wahlgren N, et al. Mechanical thrombectomy in acute ischemic stroke-patients with wake-up stroke and the elderly may benefit as well. J Stroke Cerebrovasc Dis 2016;25:2276-83.

15 Serena J, Dávalos A, Segura T, et al. Stroke on awakening: looking for a more rational management. Cerebrovasc Dis 2003;16:128-33.

16 Todo K, Moriwaki H, Saito K, et al. Early CT findings in unknown-onset and wake-up strokes. Cerebrovasc Dis 2006:21:367-71.

17 Silva GS, Lima FO, Camargo EC, et al. Wake-up stroke: clinical and neuroimaging characteristics. Cerebrovasc Dis 2010;29:336-42.

18 Bouslama M, Bowen MT, Haussen DC, et al. Selection paradigms for large vessel occlusion acute ischemic stroke endovascular therapy. Cerebrovasc Dis 2017:44:277-84.

19 Gao J, Parsons MW, Kawano H, et al. Visibility of CT Early ischemic change is significantly associated with time from stroke onset to baseline scan beyond the first 3 hours of stroke onset. J Stroke 2017;19:340-6.

20 Campbell BCV, Mitchell PJ, Kleinig TJ, et al. Endovascular therapy for ischemic stroke with perfusion-imaging selection. $N$ Engl J Med Overseas Ed 2015;372:1009-18.

21 Saver JL, Goyal M, Bonafe A, et al. Stent-retriever thrombectomy after intravenous t-PA vs. t-PA alone in stroke. N Eng/ J Med 2015;372:2285-95. 